\title{
Services for self-harm: progress and promise?
}

Nav Kapur

\section{Summary}

This editorial considers whether the quality of care for people who present to clinical services in the UK following self-harm has improved or stagnated. Some real progress has been made in the areas of service provision and research, and self-harm has never had a higher priority in policy terms. However, major gaps remain. We need to enhance people's experience of services and improve access to high-quality assessment and aftercare.

\section{Keywords}

Self-harm; suicide prevention; mental health services; liaison psychiatry; policy.

\section{Copyright and usage}

(c) The Authors, 2020. Published by Cambridge University Press on behalf of the Royal College of Psychiatrists.
Nav Kapur is Professor of Psychiatry and Population Health at the Centre for Mental Health and Safety, University of Manchester, and an honorary consultant in psychiatry with Greater Manchester Mental Health NHS Foundation Trust, UK.

Taking a long-term view of the development of mental health services can be genuinely informative, but one of the dangers with such retrospectives is that they become unduly pessimistic. House \& Owens look back over the past 25 years of self-harm services in the UK and conclude that things are simply not getting any better. ${ }^{1}$ Fifteen years ago in the BJPych I suggested areas where we might want to concentrate research and service provision efforts in order to improve our management of self-harm. ${ }^{2}$ The question is, have we been guilty of a lack of progress and 'foot-shuffling' as House \& Owens suggest, or do people who have harmed themselves and present to hospital now get better care than they did in the past?

\section{Context}

The scale of the problem is not in doubt, with presentations for selfharm making up a significant proportion of the workload of UK emergency departments and liaison psychiatry teams. It is also growing - recent studies have reported a $50 \%$ increase in selfharm among middle-aged $\mathrm{men}^{3}$ and a $70 \%$ increase among young people. ${ }^{4}$ Self-harm is a major risk factor for later suicide. House \& Owens's figure of 30000 suicide deaths linked to self-harm since 1994 represents a shocking toll, with much of this risk concentrated in the immediate aftermath of a self-harm episode. In 2019 the Multicentre Study of Self-harm in England reported that the risk of suicide after self-harm was 50 times higher than in the general population in the first year after hospital attendance, but nearly 200 times higher in the first month. ${ }^{5}$ Of course, self-harm is sometimes hidden and many people never attend hospital services. Researchers and clinicians sometimes talk about the iceberg model of self-harm, with much of the behaviour going undetected 'below the waterline', but people presenting to hospital represent the highest-risk individuals who we have an opportunity and responsibility to help.

\section{Services, research and policy}

House \& Owens lament the lack of progress in clinical service provision, accurate data and research, and guidelines and policy. I will discuss each of these areas in turn.
With respect to services, the most recent national survey on the variability in self-harm provision is now over 10 years old. ${ }^{6}$ This audit of a representative sample of hospitals found the beginnings of an improvement in the quality of self-harm services, but limited impact on the proportion of people receiving specialist assessments or follow-up. ${ }^{6}$ Mental healthcare in general hospitals has since been transformed, with a major investment in liaison psychiatry $-94 \%$ of acute hospitals now have liaison services, ${ }^{7}$ a figure that was unimaginable 25 years ago. However, whether this has improved the quality of care for people who have self-harmed remains unknown.

Considering data and research, high-quality data have been collected as part of the Multicentre Study of Self-Harm in England for over 20 years (and over 40 years in Oxford), but it is correct to say that national statistics are limited. Hospital Episode Statistics capture patients admitted to hospital after self-harm quite well, but may miss those attending emergency departments. Data sources such as the Mental Health Services Data Set and Emergency Care Data Set in England are improving and linkage to these and other databases, such as those in primary care, offers opportunities for better ascertainment. Other data linkages (for example, the Secure Anonymised Information Linkage or SAIL Databank in Wales) are already well established. Useful longitudinal data have been emerging from community surveys too. Research into self-harm has burgeoned. There have been three Cochrane reviews on the subject in recent years, ${ }^{8-10}$ with promising results for cognitive-behavioural interventions - provision of these was associated with a $30 \%$ reduction in the risk of repetition in adults. It is true, of course, that some UK research bodies have not funded trials, but the National Institute for Health Research is currently funding two large programmes that aim to develop new interventions and there are a number of smaller projects examining treatments or models of care.

In policy terms, self-harm must be seen in the wider context of suicide prevention, which has never had a higher priority. England's suicide prevention strategies in 2002 and 2012 included people who self-harm as an important 'high-risk' group, but in the 2016 revision self-harm was identified as an important area in its own right.

Strategies are all very well, but what about implementation? Selfharm is one of the three main areas in a major NHS England-funded quality improvement initiative for suicide prevention. In 2020-2021 there will also be a national Commissioning for Quality and Innovation (CQUIN) target to improve the number and quality of assessments for people who present to hospital with self-harm.

Looking forward, the National Institute for Health and Care Excellence (NICE) self-harm guidelines are being revised with an ambitious and wide-ranging scope. ${ }^{11}$ They are due to be published in 2022 and so it seems a little premature of House \& Owens to suggest that 
they will not have an impact on practice. The All Party Parliamentary Group on Suicide and Self-Harm Prevention is also launching an inquiry into the support available to young people who have selfharmed. My own reading of the NHS long-term plan is a positive one - some fundamental changes have been proposed, including integrated community services for people who have self-harmed and a comprehensive national response to patients in mental health crisis.

\section{Services for self-harm: then and now}

What about progress in the areas I highlighted in my editorial a decade and a half ago ${ }^{2}$ There was a suggestion that we make better use of observational data for research. This has been a growing area with use of novel analytic techniques such as propensity score and instrumental variable approaches. Patient involvement in training and service delivery is certainly much more prominent now, but by no means universal. Service provision has indeed developed - according to a recent UK survey the majority of liaison psychiatry teams are truly multidisciplinary. ${ }^{7}$ We may also have begun to win the battle against the unthinking use of risk tools that aim to predict outcome, although they remain a feature in many mental health services. But in other areas there has been little progress. Timely followup and aftercare is no easier to access now than then and for too many patients their experience of care remains a negative one.

\section{Where do we go from here?}

Our starting point should probably be to decide what self-harm services are for. The prompt provision of evidence-based care intended to reduce the likelihood of further self-harm while ensuring a positive patient experience would be my view. Of course, we need more research and bigger randomised trials that can tell us what works best and for whom. One neglected area is gender - very few studies consider the different treatment needs and responses of men and women. But more fundamental is a far greater emphasis on putting what we know into practice. The implementation gap for self-harm services remains substantial, particularly with respect to the provision of high-quality assessments and psychological interventions. Up until recently, no service in the UK offered routine psychological treatment to all those who had presented with self-harm, but this may now be starting to change. Understanding the rise in self-harm in young people is a priority. As well as being a major problem in its own right, the worry is that this will translate into a higher suicide rate in this group in years to come. Indeed, the incidence of suicide in young people does appear to be rising. Social media may well be one contributory factor, but self-harm is a complex behaviour and all forms of communication may have positive as well as negative effects on mental health. Social media use is primarily a societal issue, but mental health services also need to think about how best to consider this at an individual patient level. The essential role of primary care has been highlighted in research reviews and reports. Training and supervision of those on the front line is vital, as is tackling negative staff attitudes whenever we encounter them. The use of machine learning and artificial intelligence with health data in order to improve our management of self-harm should be explored further, but we need to take care that this does not simply turn into risk prediction by another name. Meaningful involvement of people with lived experience is crucial and often the best way of effecting change. We must be mindful not to exclude people who might be particularly vulnerable, such as those from lesbian, gay, bisexual and transgender (LGBT) or minority ethnic groups. Making progress in all of these areas will, of course, be more challenging in the post COVID-19 NHS, and there will be additional pressures arising from the social and economic consequences of the pandemic and its aftermath.

\section{Progress and promise?}

House \& Owens clearly take a different view of how far we have come in providing high-quality care for self-harm. They highlight service, research and policy deficiencies and call for greater investment. From where I stand, the picture isn't quite as bleak as they portray. But must we do better? Of course we must.

Nav Kapur $(\mathbb{D}$, Centre for Mental Health and Safety \& NIHR Patient Safety Translational Research Centre, University of Manchester and Greater Manchester Mental Health NHS Foundation Trust, UK

Correspondence: Nav Kapur. Email: nav.kapur@manchester.ac.uk

First received 6 Mar 2020, final revision 17 Apr 2020, accepted 8 May 2020

\section{Acknowledgements}

I thank Dr Leah Quinlivan for comments on the paper.

\section{Declaration of interest}

N.K. chaired the committees developing the NICE Guidelines for Self-Harm (Longer Term N.K. Chaired 2012 and the NICE Quality Standards on Self-Harm 2013. He is currently topic advisor for the new NICE guidelines on self-harm and Chair of the Guideline Committee for the NICE Depression Guidelines. He works with NHS England on national quality improvement initiatives for suicide and self-harm and sits on the Department of Health and Social Care's National Suicide Prevention Strategy Advisory Group for England. He is a co-investigator on the Multicentre Study of Self-Harm in England. The views expressed in this article are the author's own and not those of the Department of Health and Social Care, NHS England or NICE.

\section{References}

1 House A, Owens D. General hospital services in the UK for adults presenting after self-harm: little evidence of progress in the past 25 years. Br J Psychiatry [Epub ahead of print] 5 May 2020. Available from: https://doi.org/10.1192/bjp. 2020.85.

2 Kapur N. Management of self-harm in adults: which way now? Br J Psychiatry 2005; 187: 497-9.

3 Clements C, Hawton K, Geulayov G, Waters K, Ness J, Rehman M, et al. Selfharm in midlife: Analysis using data from the Multicentre Study of Self-harm in England. Br J Psychiatry 2019; 215: 600-7.

4 Morgan C, Webb RT, Carr MJ, Kontopantelis E, Green J, Chew-Graham CA, et al. Incidence, clinical management, and mortality risk following self harm among children and adolescents: cohort study in primary care. BMJ 2017; 359: j4351.

5 Geulayov G, Casey D, Bale L, Brand F, Clements C, Farooq B, et al. Suicide following presentation to hospital for non-fatal self-harm in the Multicentre Study of Self-harm: a long-term follow-up study. Lancet Psychiatry 2019; 6: 1021-30.

6 Cooper J, Steeg S, Bennewith O, Lowe M, Gunnell D, House A, et al. Are hospital services for self-harm getting better? An observational study examining management, service provision and temporal trends in England. BMJ Open 2013; 3: e003444.

7 Walker A, Barrett JR, Lee W, West RM, Guthrie E, Trigwell P, et al. Organisation and delivery of liaison psychiatry services in general hospitals in England: results of a national survey. BMJ Open 2018; 8: e023091.

8 Hawton K, Witt KG, Taylor Salisbury TL, Arensman E, Gunnell D, Hazell P, et al. Psychosocial interventions for self-harm in adults. Cochrane Database of Systematic Reviews 2016; Issue 5: CD012189. Available from: https://doi.org/ 10.1002/14651858.CD012189.

9 Hawton K, Witt KG, Taylor Salisbury TL, Arensman E, Gunnell D, Hazell P, et al. Pharmacological interventions for self-harm in adults. Cochrane Database of Systematic Reviews 2015; Issue 7: CD011777. Available from: https://doi.org/ 10.1002/14651858.CD011777.

10 Hawton K, Witt KG, Taylor Salisbury TL, Arensman E, Gunnell D, Townsend E, et al. Interventions for self-harm in children and adolescents. Cochrane Database of Systematic Reviews 2015; Issue 12: CD012013. Available from: https://doi.org/10.1002/14651858.CD012013.

11 National Institute for Health and Care Excellence. Guideline Scope. Self-Harm: Assessment, Management and Preventing Recurrence. NICE, 2019 (https:// www.nice.org.uk/guidance/gid-ng10148/documents/final-scope). 